\title{
Comparative effectiveness and safety of anticoagulants for the treatment of heparin-induced thrombocytopenia
}

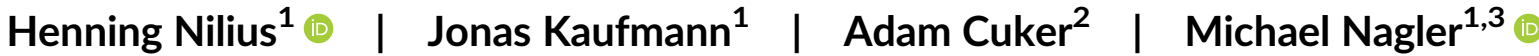

${ }^{1}$ Department of Clinical Chemistry, Inselspital, Bern University Hospital, Bern, Switzerland

${ }^{2}$ Department of Medicine and Department of Pathology and Laboratory Medicine, University of Pennsylvania Perelman School of Medicine, Philadelphia, Pennsylvania, USA

${ }^{3}$ University of Bern, Bern, Switzerland

\section{Correspondence}

Michael Nagler, Inselspital, Bern University Hospital, and University of Bern, 3010 Bern, Switzerland.

Email: michael.nagler@insel.ch

\begin{abstract}
Background: The effectiveness and safety of non-heparin anticoagulants for the treatment of heparin-induced thrombocytopenia (HIT) are not fully established, and the optimal treatment strategy is unknown. In a systematic review and meta-analysis, we aimed to determine precise rates of platelet recovery, new or progressive thromboembolism (TE), major bleeding, and death for all non-heparin anticoagulants and to study potential sources of variability.

Methods: Following a detailed protocol (PROSPERO: CRD42020219027), EMBASE and Medline were searched for all studies reporting clinical outcomes of patients treated with non-heparin anticoagulants (argatroban, danaparoid, fondaparinux, direct oral anticoagulants [DOAC], bivalirudin, and other hirudins) for acute HIT. Proportions of patients with the outcomes of interest were pooled using a randomeffects model for each drug. The influence of the patient population, the diagnostic test used, the study design, and the type of article was assessed.

Results: Out of 3194 articles screened, 92 studies with 119 treatment groups describing 4698 patients were included. The pooled rates of platelet recovery ranged from $74 \%$ (bivalirudin) to $99 \%$ (fondaparinux), TE from 1\% (fondaparinux) to $7 \%$ (danaparoid), major bleeding from $1 \%$ (DOAC) to $14 \%$ (bivalirudin), and death from $7 \%$ (fondaparinux) to $19 \%$ (bivalirudin). Confidence intervals were mostly overlapping, and results were not influenced by patient population, diagnostic test used, study design, or type of article.

Discussion: Effectiveness and safety outcomes were similar among various anticoagulants, and significant factors affecting these outcomes were not identified. These findings support fondaparinux and DOACs as viable alternatives to conventional anticoagulants for treatment of acute HIT in clinical practice.
\end{abstract}

\section{1 | INTRODUCTION}

Heparin-induced thrombocytopenia (HIT) still affects a large number of patients. An estimated 12 million hospitalized patients receive

Henning Nilius and Jonas Kaufmann contributed equally to this study. heparin derivatives every year in the USA; approximately one in 40 patients receiving unfractionated heparin (UFH) and one in 500 patients receiving low molecular weight heparin (LMWH) develops HIT. ${ }^{1-3}$ HIT is regarded as one of the most prothrombotic clinical states with a high risk (at least 50\%) of thromboembolism (TE) and even death. ${ }^{4,5}$ Despite this, the effectiveness and safety of 
anticoagulants for the treatment of HIT are not fully established, and the optimal treatment strategy is still unknown. ${ }^{6}$

Intravenous anticoagulants such as argatroban and bivalirudin are conventional treatments for acute HIT, which are licensed in various countries. The downsides of these drugs, however, are important: the bleeding risk is high, a constant intravenous line and laborious laboratory monitoring are required, and they are expensive and therefore not available in many health care settings. ${ }^{7,8}$ Fondaparinux and direct oral anticoagulants (DOACs), which have emerged as potential alternatives to intravenous anticoagulants in recent years, avoid these problems. ${ }^{9,10}$ Their effectiveness and safety is, however, not as wellestablished, and they are recommended in less severely ill patients only. ${ }^{6}$

The comparative effectiveness and safety of anticoagulants for the treatment of HIT is unclear because adequately designed clinical trials are lacking. Evidence is largely limited to observational studies, which in turn, are associated with important methodological limitations: small sample size; not all important outcomes were studied; control groups receiving other anticoagulants were mostly missing; and potential sources of variability such as patient population, diagnostic testing strategy, and study design might have influenced the results.

To fill this critical gap in knowledge, we conducted a systematic review and meta-analysis aiming (1) to retrieve all available data from studies observing patients treated with various non-heparin anticoagulants for acute HIT, (2) to perform a quantitative meta-analysis for important clinical outcomes (platelet recovery, TE, major bleeding, and death), and (3) to study potential sources of variability (patient population, diagnostic testing strategy, study design, and type of publication). These data will help to appraise the effectiveness and safety of various non-heparin anticoagulants, even in the absence of adequately designed randomized controlled trials.

\section{2 | MATERIAL AND METHODS}

\section{1 | Protocol, study identification; and screening}

A study protocol was submitted to the PROSPERO international prospective register of systematic reviews (CRD 42020219027). A sensitive search strategy was developed to identify all studies which assessed the effectiveness and safety outcomes of anticoagulants used for the treatment of acute HIT. We searched MEDLINE and EMBASE through the Ovid platform from inception until November 10, 2020. The following search terms were used: (heparin-induced thrombocytopenia.tw OR heparin induced thrombocytopenia.tw) AND (Hirudins[MeSH OR hirudins. tw OR rivaroxaban[MeSH] OR rivaroxaban.tw OR Dabigatran[MeSH] OR Dabigatran.tw OR Danaparoid.tw OR lepirudin.tw OR argatroban.tw OR Fondaparinux.tw OR Bivalirudin.tw OR Desirudin.tw OR Apixaban. tw OR Edoxaban.tw). The search was limited to studies in humans; no restrictions were applied concerning language or type of publication. Additionally, references of articles were manually checked for potentially eligible studies. Records were screened in duplicate by two investigators
(J.K., M.N.) and duplicates were removed. The manuscript was prepared following the Preferred Reporting Items for Systematic Reviews and Meta-Analysis (PRISMA) guideline. ${ }^{11}$

\section{2 | Study eligibility}

The eligibility of studies was assessed in full-text by two investigators (J.K., M.N.) and disputes were resolved by discussion. The following inclusion criteria were applied: (1) treatment of acute HIT with one of the specified non-heparin anticoagulants mentioned below, and (2) reporting of at least one of the following outcomes: (a) platelet recovery, (b) new or progressive TE, (c) major bleeding, or (d) death. Exclusion criteria were: (1) review articles without new data, (2) insufficient clinical data, (3) double publications, (4) case reports, and (5) investigational therapies other than the pre-specified anticoagulants. We did not apply exclusion criteria regarding study design, type of publication, publication date, or language.

\section{3 | Definition of drugs, outcomes, and other variables of interest}

The following categories of treatment schemes were defined: argatroban, danaparoid, fondaparinux, DOACs, bivalirudin, and other hirudins. The DOACs included apixaban, edoxaban, dabigatran, and rivaroxaban. Other hirudins included lepirudin, desirudin, and hirudin. In cases where patients were treated with more than one non-heparin anticoagulant, only the drug administered for the longest duration was considered for analysis.

Acute HIT was defined as newly diagnosed HIT prior to platelet recovery. Platelet recovery and new or progressive TE were defined as effectiveness outcomes, and major bleeding and death of any cause were defined as safety outcomes. Platelet recovery was defined as an increase in platelet count of $\geq 100 \times 10^{9} / \mathrm{L}$ or doubling of the nadir platelet count, or a $30 \%$ increase from the nadir if the nadir was above $100 \times 10^{9} /$ L. $^{12}$ TE was defined as a new objectively verified arterial or venous TE or progression of already present TE. Major bleeding was defined according to the classification of the International Society on Thrombosis and Hemostasis (ISTH). ${ }^{13}$ If bleeding events were reported using the Common Terminology Criteria for Adverse Events (CTCAE) of the US National Cancer Institute, bleeding grade 3 and 4 were defined as major bleeding. ${ }^{14}$ All events that were observed within the observation time defined by the primary study were counted.

Anticipating that factors related to the study design of the primary studies might have affected the outcomes, we defined several potential sources of variability for sensitivity analyses. First, different strategies to diagnose HIT were addressed by creating the following groups of studies: (1) clinically suspected HIT, (2) HIT diagnosed using heparin-platelet-factor-4 antibody tests (H/PF4), and (3) HIT diagnosed by a washed platelet assay (serotonin release assay, SRA, or heparin-induced platelet-aggregation assay, HIPA). Second, different 
study populations were considered by grouping studies into (a) patients with HIT complicated by thrombosis at inclusion (HITT), (b) patients with isolated HIT without thrombosis (HIT), and (c) mixed patients. The study design was categorized into (a) prospective observational studies (including single-arm interventional studies), (b) retrospective observational studies, and (c) randomized controlled trials (RCTs). Article types were classified as either (a) journal articles (published in a peer-reviewed scientific journal), or (b) congress abstracts.

\section{4 | Data extraction}

The following data were extracted by two investigators in parallel (J.K., M.N.): first author, year of publication, type of publication, study design, number of participants, the population of participants, the diagnostic test used, age, drug, observation time, number of patients included in each treatment group, the number of patients with (a) platelet recovery, (b) new or progressive TE, (c) major bleeding, and (d) death. Data were collected per treatment group. The extracted data were exported to a spreadsheet and checked for errors by a third author (H.N.).

\subsection{Assessment of methodological quality and risk of bias}

The quality of the primary articles and the risk of bias were independently assessed by J.K.,H.N. and M.N. using an adaptation of the Newcastle-Ottawa-Scale (NOS). ${ }^{15}$ The NOS is an established tool for the assessment of the risk of bias in non-randomized observational studies, which was adapted to fit our research question. Three different domains were assessed with several signaling questions. Points were assigned for each domain as follows: selection of

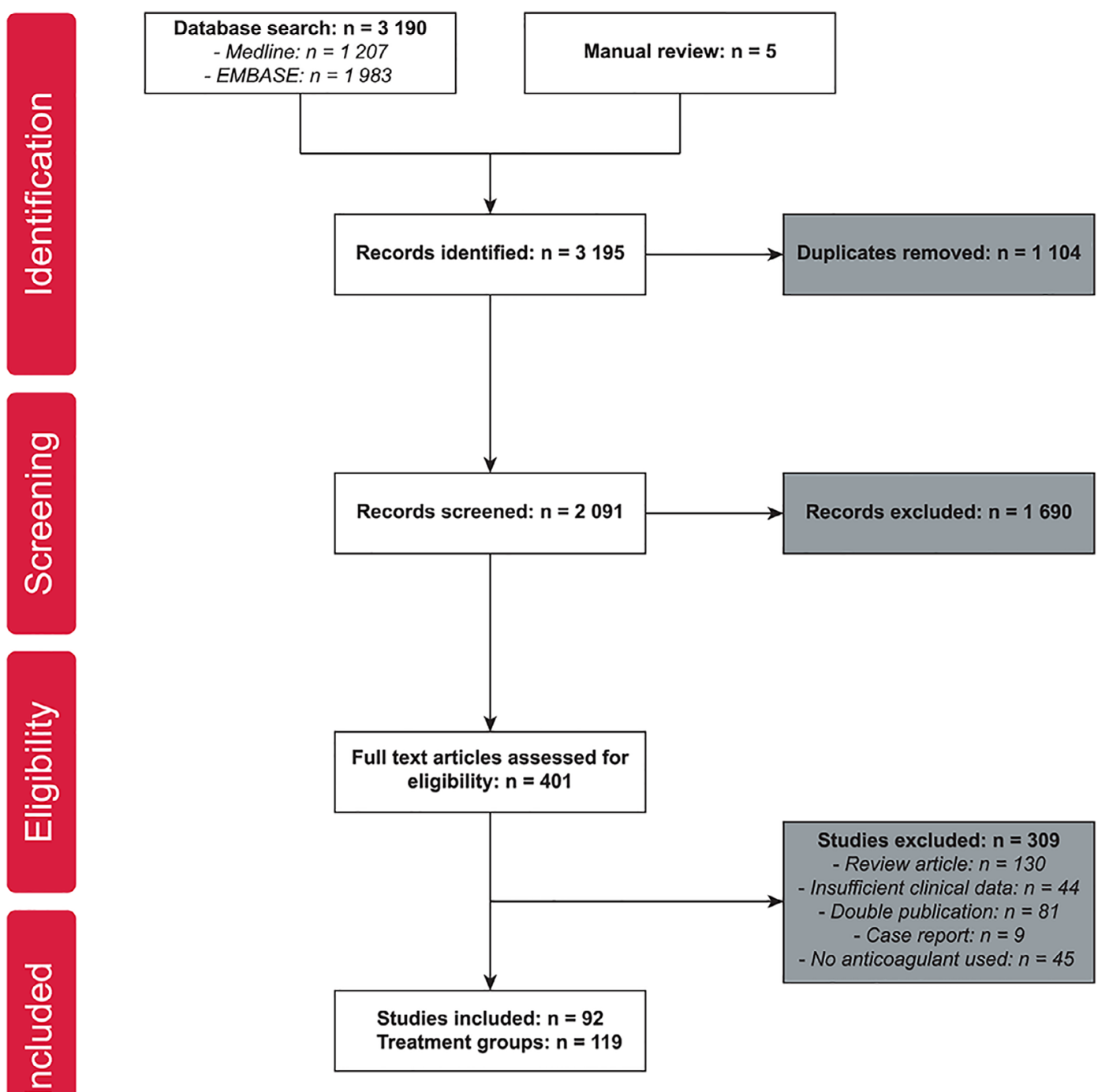

FIGURE 1 Flow of studies [Color figure can be viewed at wileyonlinelibrary.com] 
patients (up to three points), comparability between study groups (up to two points), and outcome measures (up to three points) Studies with a score of $\geq 6$ points, $3-5$ points, and $\leq 2$ points were considered low risk of bias, medium risk of bias, and high risk of bias, respectively. If the investigators did not agree, disputes were resolved by discussion. The adapted NOS template is reported in the supplementary material. Contour-enhanced funnel plots were created for each of the outcomes to assess publication bias.

\subsection{Synthesis of data}

Statistical analyses were done with the "meta" and "metafor" package for " $R$ ". ${ }^{16-18}$ The principal summary measure considered for this metaanalysis was the proportion of patients with the outcomes of interest (platelet recovery, TE, major bleeding, and death). The logittransformed proportions were pooled using a random-effects model based on a random intercept logistic regression model. A randomeffect model was chosen since we expected high heterogeneity among the studies. We decided against a Freeman-Tukey double arcsine transformation to avoid misleading results due to differences in study size. ${ }^{19}$ Corresponding $95 \%$ confidence intervals $(\mathrm{Cl})$ were calculated. The proportions and corresponding $95 \% \mathrm{Cl}$ were reported back-transformed. Heterogeneity between studies was tested using Higgins $I^{2}$. We constructed forest plots displaying the pooled percentage for each of the outcomes. To explore potential sources of variability and bias, we pooled proportions separately for each level of the variable and created forest plots. $95 \% \mathrm{Cl}$ and Higgins $\mathrm{I}^{2}$ were also calculated for each of the levels.

\section{3 | RESULTS}

\section{1 $\quad$ Study identification and selection}

The literature search identified 3190 records (MEDLINE: $n=1207$; EMBASE: $n=1983$ ) and an additional five were found after a manual search of the bibliographies of eligible studies. After removing duplicates, 2091 records were screened. One-thousand six-hundred ninety articles were excluded because they did not meet the inclusion criteria or focused on an unrelated topic. Four-hundred-and-one articles were assessed in full-text. Of these, 309 were excluded because no new data were given ( $n=130$ ), insufficient clinical data reported $(n=44)$, double publication ( $n=81$ ), case reports only $(n=9)$, or used an investigational therapy other than one of the pre-specified anticoagulants $(n=45)$. Ninety-two articles reporting on 4698 patients in 119 study groups were eventually included. ${ }^{20-111} \mathrm{~A}$ flow-diagram of the articles is illustrated in Figure 1.

\section{2 | Study characteristics and patients}

The study design was prospective in 12 publications, retrospective in 78 publications, and a RCT was conducted in two cases. One-hundred and nineteen treatment groups were identified. Seventeen studies were published as congress abstracts and 75 as journal articles. The number of participants ranged from two to 697 and the publication date between 1995 and 2020. The observation time ranged from a few days to 5 years after discharge. Platelet recovery was reported in 63 treatment groups, TE in 101, major bleeding in 96, and death in 100. The mean age of the participants ranged from 5 months to 73.7 years. HIT was diagnosed
Are reported patients representative for all treated patients

Selection of the control group

Were patients really treated

Were endpoints absent at the time of inclusion

Are exposed and unexposed patients comparable

Determination of endpoints

Follow up long enough

Complete follow up
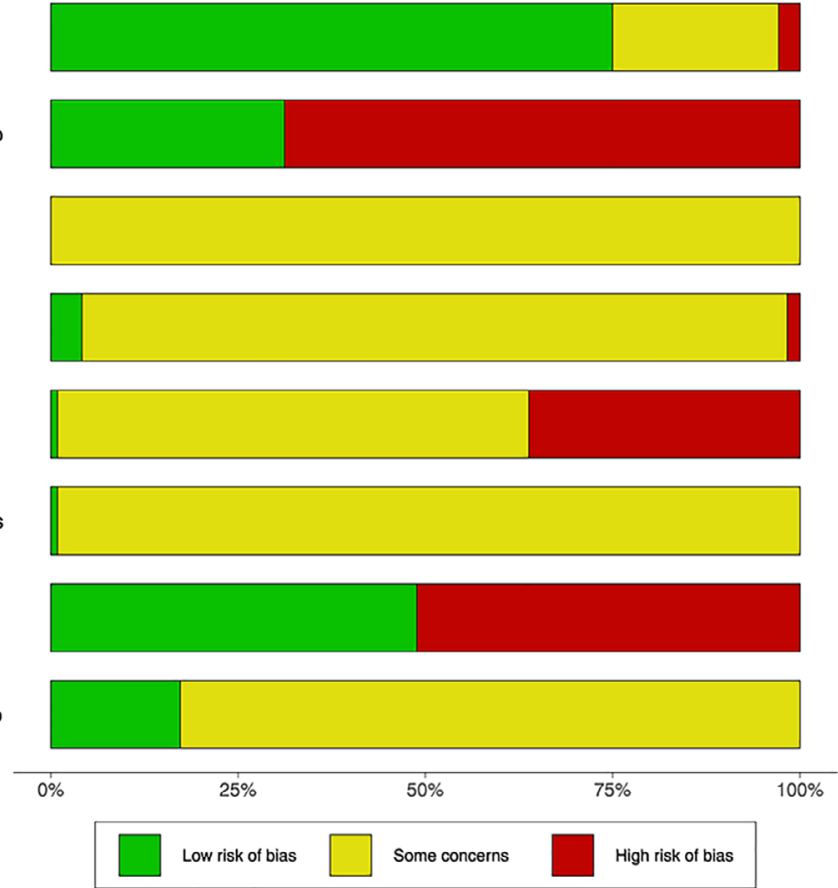

FIGURE 2 Summary of the risk of bias based on an adaptation of the Newcastle-Ottawa-Scale [Color figure can be viewed at wileyonlinelibrary.com] 
using a washed platelet assay (SRA/HIPA) in 20 treatment groups, a heparin/PF4 antibody assay in 42 treatment groups, and clinical characteristics alone in 57 groups. The study population consisted of patients with thrombosis in 16 treatment groups, patients without thrombosis in 13 treatment groups, mixed patients in 67 groups, and not specified in 23 treatment groups. Argatroban was used in 39 groups, danaparoid in 19 groups, fondaparinux in 19 groups, DOACs in eight groups, bivalirudin in 13 groups, and other hirudins in 21 groups. Out of eight groups utilizing DOACs, rivaroxaban was used in five groups, apixaban in two groups, and dabigatran in one group. Detailed study characteristics are given in Table S1 of the supplementary material.

\section{3 | Methodological quality}

The risk of bias was high in 66 out of 91 studies according to the adapted NOS score. It was medium in 25 studies and low in only one study. Common study limitations included lack of confirmation of whether patients received the treatment, which was prescribed, lack of appropriate control groups, and short follow-up period. A summary plot of the risk of bias is displayed in Figure 2 and a detailed traffic light plot can be seen in Figure S2 (supplementary material). Contourenhanced funnel plots are given in Figure S3; asymmetry is present on visual inspection of each of the outcomes.

\section{4 | Effectiveness: platelet recovery and new or progressive TE}

The pooled rates of platelet recovery for each of the drugs are illustrated in Figure 3(A). They ranged from 0.74 with bivalirudin $(95 \% \mathrm{Cl}$ : $0.58,0.85 ; 1^{2}=90.3 \%$; based on 509 patients) to 0.99 with fondaparinux (95\% Cl: $\left.0.90,1.00 ; \mathrm{I}^{2}=44.7 \%, n=351\right)$. The pooled

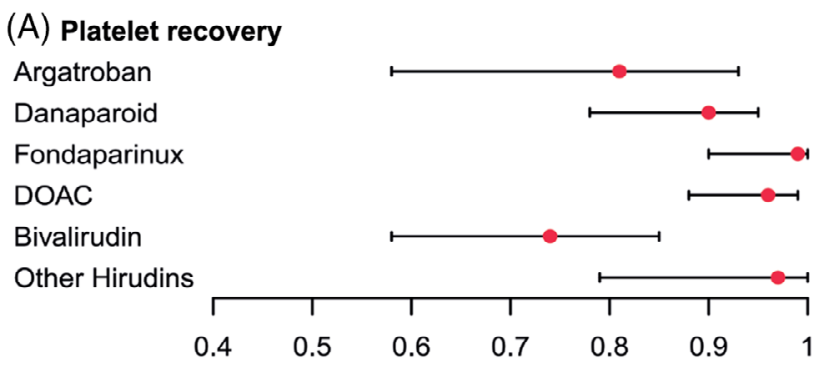

$\begin{array}{cccc}\text { Proportion } & \mathbf{9 5 \%} \mathbf{C l} & \text { Groups } & \mathbf{n} \\ 0.81 & {[0.58,0.93]} & 13 & 247 \\ 0.90 & {[0.78,0.95]} & 15 & 359 \\ 0.99 & {[0.90,1.00]} & 14 & 351 \\ 0.96 & {[0.88,0.99]} & 5 & 74 \\ 0.74 & {[0.58,0.85]} & 4 & 509 \\ 0.97 & {[0.79,1.00]} & 12 & 144\end{array}$

(B) Thromboembolic event

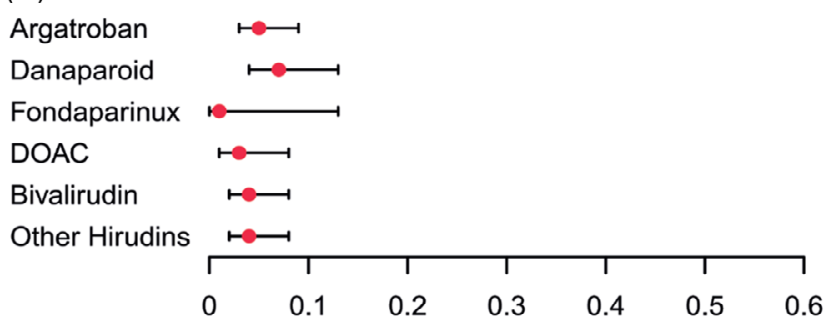

$\begin{array}{cccc}\text { Proportion } & \mathbf{9 5 \%} \mathbf{~ C l} & \text { Groups } & \mathbf{n} \\ 0.05 & {[0.03,0.09]} & 33 & 1733 \\ 0.07 & {[0.04,0.13]} & 15 & 506 \\ 0.01 & {[0.00,0.13]} & 15 & 241 \\ 0.03 & {[0.01,0.08]} & 8 & 124 \\ 0.04 & {[0.02,0.08]} & 8 & 688 \\ 0.04 & {[0.02,0.08]} & 17 & 788\end{array}$

(C) Major Bleeding

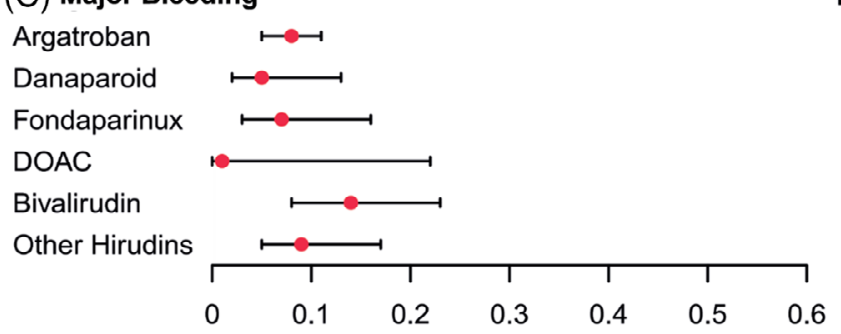

$\begin{array}{cccc}\text { Proportion } & \mathbf{9 5 \%} \mathbf{~ C l} & \text { Groups } & \mathbf{n} \\ 0.08 & {[0.05,0.11]} & 34 & 1100 \\ 0.05 & {[0.02,0.13]} & 17 & 551 \\ 0.07 & {[0.03,0.16]} & 14 & 355 \\ 0.01 & {[0.00,0.22]} & 8 & 124 \\ 0.14 & {[0.08,0.23]} & 11 & 762 \\ 0.09 & {[0.05,0.17]} & 17 & 772\end{array}$

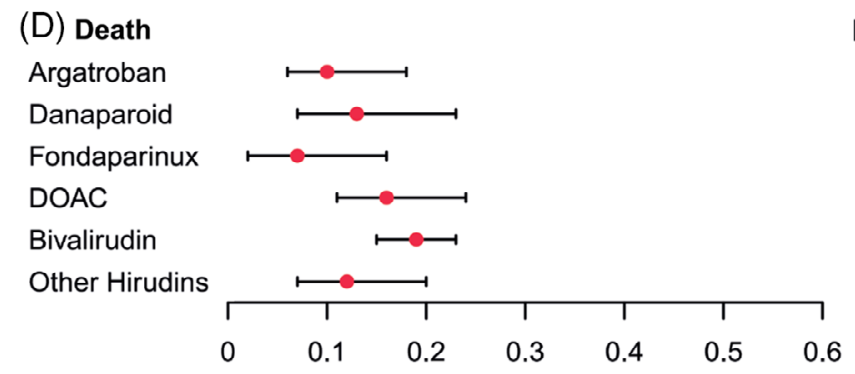

$\begin{array}{cccc}\text { Proportion } & \mathbf{9 5 \%} \mathbf{~ C l} & \text { Groups } & \mathbf{n} \\ 0.10 & {[0.06,0.18]} & 30 & 1573 \\ 0.13 & {[0.07,0.23]} & 19 & 602 \\ 0.07 & {[0.02,0.16]} & 17 & 266 \\ 0.16 & {[0.11,0.24]} & 8 & 124 \\ 0.19 & {[0.15,0.23]} & 10 & 818 \\ 0.12 & {[0.07,0.20]} & 16 & 707\end{array}$

FIGURE 3 Effectiveness and safety outcomes of various anticoagulants used in the treatment of HIT. Pooled proportions of (A) platelet recovery, (B) thromboembolism, (C) major bleeding, and (D) death are shown according to the treatment scheme. The logit-transformed proportions were pooled using a random-effects model based on a random intercept logistic regression model. Groups, number of treatment groups; $95 \% \mathrm{Cl}$, $95 \%$ confidence interval; $n$, number of patients [Color figure can be viewed at wileyonlinelibrary.com] 


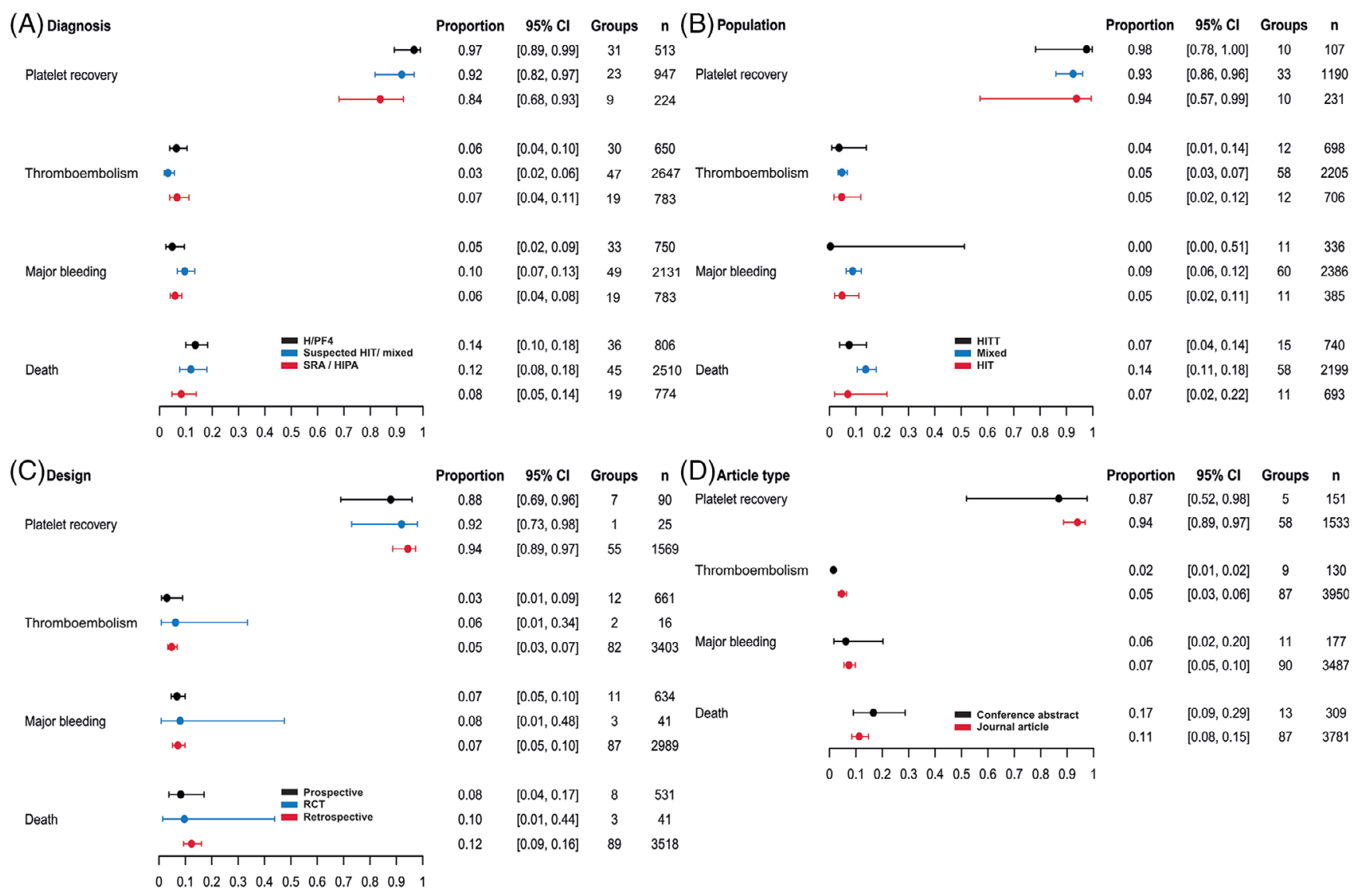

FIGURE 4 Potential sources of variability in studies investigating anticoagulants for the treatment of HIT. Pooled proportions of key outcomes according to (A) diagnostic reference standard, (B) characteristics of patient population, (C) study design, and (D) type of publication are shown. The logit-transformed proportions were pooled using a random-effects model based on a random intercept logistic regression model.

Groups, number of treatment groups; $95 \% \mathrm{Cl}, 95 \%$ confidence interval; $\mathrm{n}$, number of patients [Color figure can be viewed at wileyonlinelibrary.com]

platelet recovery rates were $0.81\left(95 \% \mathrm{Cl}: 0.58,0.93 ; \mathrm{I}^{2}=41.8 \%\right.$, $n=247)$ for argatroban, $0.90\left(95 \% \mathrm{Cl}: 0.78,0.95 ; \mathrm{I}^{2}=60.9 \%\right.$, $n=359)$ for danaparoid, 0.96 for DOACs $(95 \% \mathrm{Cl}: 0.88,0.99$; $\left.\mathrm{I}^{2}=0.0 \%, n=74\right)$, and 0.97 (95\% Cl: $\left.0.79,1.00, \mathrm{I}^{2}=0.00 \%, n=144\right)$ for other hirudins. Cl's were overlapping for most drugs. The $\mathrm{Cl}$ for bivalirudin, however, did not overlap with the $\mathrm{Cl}$ for fondaparinux or DOACs.

The TE rates are displayed for all drugs in Figure 3(B). Even though rates ranged from $0.01\left(95 \% \mathrm{Cl}: 0.00,0.13 ; 1^{2}=0.00 \%\right.$, $n=241)$ with fondaparinux to 0.07 (95\% Cl: $0.04,0.13 ; I^{2}=2.88 \%$, $n=506)$ with danaparoid, Cls were largely overlapping. The rate was 0.05 (95\% Cl: $0.03,0.09 ; \mathrm{I}^{2}=35.27 \%, n=1733$ ) with argatroban, 0.03 (95\% Cl: $\left.0.01,0.08 ; I^{2}=0.0 \%, n=124\right)$ with DOACs, $0.04(95 \%$ Cl: $\left.0.02,0.08 ; I^{2}=31.7 \%, n=688\right)$ with bivalirudin, and $0.04(95 \%$ Cl: $0.02,0.08 ; 1^{2}=32.5 \%, n=788$ ) with other hirudins.

\section{5 | Safety: major bleedings and deaths}

Pooled proportions of patients with major bleeding are illustrated in Figure $3(\mathrm{C})$. The range of proportions was 0.01 (95\% Cl: $0.00,0.22$; $\left.\mathrm{I}^{2}=0.00 \%, n=124\right)$ for DOACs to $0.14(95 \% \mathrm{Cl}: 0.08,0.23$;
$\left.\mathrm{I}^{2}=88.1 \%, n=762\right)$ for bivalirudin. The rates were $0.08(95 \% \mathrm{Cl}$ : $\left.0.05,0.11 ; I^{2}=44.6 \%, n=1100\right)$ for argatroban, 0.05 (95\% Cl: 0.02 , $0.14 ; 1^{2}=70.7 \%, n=551$ ) for danaparoid, 0.07 for fondaparinux (95\% Cl: $0.03,0.16 ; I^{2}=28.2 \%, n=355$ ), and 0.09 (95\% Cl: 0.05 , $0.17 ; I^{2}=68.5 \%, n=772$ ) for other hirudins. The Cl's were overlapping for all drugs.

The pooled death rates for each of the anticoagulants are displayed in Figure 3(D). They ranged from 0.07 (95\% Cl: 0.02, 0.16; $\left.\mathrm{I}^{2}=24.4 \%, n=266\right)$ with fondaparinux to 0.19 (95\% Cl: $0.15,0.23$; $\mathrm{I}^{2}=40.3 \%, n=818$ ) with bivalirudin. They were 0.10 with argatroban (95\% Cl: $0.06,0.18 ; I^{2}=73.7 \%, n=1573$ ), 0.13 with danaparoid (95\% Cl: $\left.0.07,0.23 ; I^{2}=73.6 \%, n=602\right), 0.16$ with DOACs $(95 \% \mathrm{Cl}$ : $\left.0.11,0.24 ; I^{2}=0.00 \%, n=124\right)$, and 0.12 (95\% Cl: $0.07,0.20$; $\left.\mathrm{I}^{2}=71.3 \%, n=707\right)$ with other hirudins. The Cls of the drugs were overlapping.

\subsection{Sources of variability}

Hypothesizing that characteristics of study design, patient population, diagnostic tests, and type of publication might have influenced the results, we conducted sensitivity analyses with respect to these 
variables. The type of diagnostic test (SRA/ HIPA vs H/PF4 immunoassay vs clinical criteria) did not affect the proportions of platelet recovery, TE recurrence, major bleeding, or death (Figure $4(\mathrm{~A})$ ); $\mathrm{Cl}$ measures were widely overlapping. The study population (patients with TE vs without TE vs mixed patients) did not influence the results of the outcomes and $\mathrm{Cl}$ measures were overlapping (Figure 4(B)). In the case of the study design used (prospective vs retrospective vs $\mathrm{RCT}$ ), no apparent differences were found (Figure $4(\mathrm{C})$ ). The $\mathrm{Cl}$ of the RCT group was wide since this group included the lowest number of studies and patients. The article type (journal article vs. conference abstract) showed widely overlapping $\mathrm{Cl}$ as well (Figure 4(D)).

\section{4 | DISCUSSION}

In a comprehensive systematic review and meta-analysis, we (a) summarized data of 119 study groups reporting on 4698 patients treated with various non-heparin anticoagulants for acute HIT, (b) performed quantitative meta-analyses of key clinical outcomes (platelet recovery, TE, major bleeding, and death), and (c) studied the influence of potential sources of variability (patient population, diagnostic testing strategies, study design, and type of publication). The rates of platelet recovery, new or progressive TE, major bleeding, and death were mostly similar between drugs. Fondaparinux and DOACs appear to be equally safe and effective compared to intravenous anticoagulants. These findings were not affected by (a) patient populations, (b) diagnostic testing strategies, (c) the study design, or (d) type of publication.

Few previous publications summarized observational data on the treatment of acute HIT. Sun et al. included nine studies with 689 participants comparing argatroban to bivalirudin and lepirudin, and observing similar numbers of bleeding events and TE. ${ }^{112}$ Bhatt et al. pooled the data of 43 patients treated with fondaparinux for acute HIT following cardiovascular interventions and reported TE recurrence estimates (5\%) and bleeding (7\%). ${ }^{113}$ Similar results were found in a broader meta-analysis of fondaparinux by Linkins et al. including nine studies and 118 patients. ${ }^{9}$ Estimates for DOAC-treated patients were calculated in a meta-analysis by Shatzel et al. ( $n=54)$; however, $78 \%$ were initially treated with parenteral anticoagulants. A bleeding rate of $5.5 \%$ and no deaths were reported. ${ }^{114}$

Our investigation has several strengths, which are in contrast to previous publications mentioned above. First, we conducted a comprehensive literature search, including all studies available without restrictions on population, type of article, publication date, or language. Secondly, all non-heparin anticoagulants used for the treatment of acute HIT were considered. Thirdly, quantitative meta-analyses of important clinical outcomes were conducted (platelet recovery, new or progressive TE, major bleeding, and death). Fourthly, we studied the influence of potential sources of variability (patient population, diagnostic testing strategy, study design, and type of publication).

Several limitations appear; most of them are inherent issues to any meta-analytic approach. First, our analysis relies on data obtained in various primary studies and the methodology of these studies is limited (e.g., small studies without control groups). Adequately designed randomized controlled trials are not available and the risk of bias was low in one study only. Excluding all other studies would make any meta-analysis and meaningful interpretation impossible. As long as high-quality data are not available, we aimed to summarize and pool all published clinical data, thus supporting clinical decisionmaking and development of future treatment guidelines. Following current recommendations, ${ }^{115}$ we addressed the possible risk of bias due to the primary studies' methodological limitations by conducting several sensitivity analyses. We repeated the analysis in studies (a) with a more accurate testing strategy (SRA/HIPA), (b) different patient populations, (c) study designs, and (d) study population without identifying major differences. However, we cannot fully exclude that this might have introduced any bias. Secondly, we were not able to conduct a sensitivity analysis considering differences in observation time among studies. It varied substantially among publications and was not reported precisely in many studies (e.g., "hospital stay"). We cannot exclude that differences in observation times among different drugs might have introduced a risk of bias. Thirdly, contour-enhanced funnel plots showed asymmetry, suggesting a potential underreporting of unfavorable results in small studies. However, it would have affected small studies, only marginally contributing to the overall estimates. Fourthly, various dosing schemes were reported for the same drug but establishing dose-response relationships were outside the scope of this work. Fifthly, we grouped all DOACs together because few studies were available; thus we cannot exclude that there may be differences in effectiveness and safety among different DOACs. Finally, a certain degree of selection bias might be present. Considering that fondaparinux and DOACs could have been given to a less ill patient population, resulting in more favorable outcomes for these drugs. Likewise, if sicker patients received drugs like bivalirudin or argatroban, this could bias the results against these agents.

Even though the level of evidence is sparse for some of the drugs (e.g., argatroban), the results of the current systematic review and meta-analysis suggest that there are no major differences among nonheparin anticoagulants for treatment of acute HIT with respect to safety and effectiveness. These findings support fondaparinux and DOACs as viable alternatives to conventional anticoagulants for treatment of acute HIT in clinical practice. Fondaparinux and DOACs are cost-effective, easy-to-manage, and potentially safer than intravenous anticoagulants. ${ }^{10}$ Ideally, this hypothesis should be tested in an adequately designed RCT. However, given that conducting RCTs in patients with HIT remains extremely difficult, ${ }^{116}$ our results represent the best level of evidence available. Future high-quality observational studies will improve the findings of meta-analyses and we encourage investigators of observational studies to conduct longer follow-up, refine the measurements of clinical outcomes, and improve reporting of results.

In conclusion, pooling data from 119 treatment groups and 4698 patients to estimate important clinical outcomes (platelet recovery, TE, major bleeding, death), we did not identify major differences among non-heparin anticoagulants for the treatment of acute HIT. These findings were not affected by the patient population, diagnostic 
testing strategies, study design, or type of publication. Our results support fondaparinux and DOACs as viable alternatives to conventional agents for the treatment of acute HIT in clinical practice. In the absence of adequately designed RCTs, these findings represent the best level of evidence available.

\section{ACKNOWLEDGMENTS}

This study was supported by a research grant of the Swiss National Science Foundation (\#179334).

\section{CONFLICT OF INTEREST}

Conflict-of-interest disclosure: Michael Nagler received research grants from Bayer Healthcare. Adam Cuker has served as a consultant for synergy and his institution has received research support on his behalf from alexion, Bayer, Novartis, novo Nordisk, Pfizer, Sanofi, spark, and Takeda. All other authors declare that no conflict of interest exists.

\section{AUTHOR CONTRIBUTIONS}

Henning Nilius collected data, wrote the analysis plan, performed the statistical analysis, and wrote the manuscript; Jonas Kaufmann performed the literature search, and collected the data; Adam Cuker contributed to study design, analysis of the data, and revised the manuscript; Michael Nagler designed the study, contributed to the literature search and data collection, analyzed the data, and wrote the manuscript.

\section{DATA AVAILABILITY STATEMENT}

The data that support the findings of this study are available from the corresponding author upon reasonable request.

\section{ORCID}

Henning Nilius (D) https://orcid.org/0000-0002-1323-3116

Michael Nagler (DD https://orcid.org/0000-0003-4319-2367

\section{REFERENCES}

1. American Hospital Association. 2020 AHA Hospital Statistics. Chicago, IL: AHA Resource Center; 2020:1-4.

2. Rice L. Heparin-induced thrombocytopenia: myths and misconceptions (that will cause trouble for you and your patient). Arch Intern Med. 2004;164:1961-1964.

3. Martel N, Lee J, Wells PS. Risk for heparin-induced thrombocytopenia with unfractionated and low-molecular-weight heparin thromboprophylaxis: a meta-analysis. Blood. 2005;106:2710-2715.

4. Greinacher A. CLINICAL PRACTICE. Heparin-induced thrombocytopenia. N Engl J Med. 2015;373:252-261.

5. Warkentin T. Heparin-induced thrombocytopenia in critically ill patients. Semin Thromb Hemost. 2015;41:49-60.

6. Cuker A, Arepally GM, Chong BH, et al. American Society of Hematology 2018 guidelines for management of venous thromboembolism: heparin-induced thrombocytopenia. Blood Adv. 2018;2:33603392.

7. Garcia DA, Baglin TP, Weitz JI, Samama MM. Parenteral anticoagulants. Chest. 2012;141:e24S-e43S.

8. Patrick AR, Winkelmayer WC, Avorn J, Fischer MA. Strategies for the management of suspected heparin-induced thrombocytopenia. Pharmacoeconomics. 2007;25:949-961.
9. Linkins L-A, Hu G, Warkentin TE. Systematic review of fondaparinux for heparin-induced thrombocytopenia: when there are no randomized controlled trials. Res Pract Thromb Haemost. 2018;2:678-683.

10. Aljabri A, Huckleberry $\mathrm{Y}$, Karnes JH, et al. Cost-effectiveness of anticoagulants for suspected heparin-induced thrombocytopenia in the United States. Blood. 2016;128:3043-3051.

11. Moher D, Liberati A, Tetzlaff J, Altman DG. Preferred reporting items for systematic reviews and meta-analyses: the PRISMA statement. BMJ. 2009;339:b2535-b2535.

12. Ahmed I, Majeed A, Powell R. Heparin induced thrombocytopenia: diagnosis and management update. Postgrad Med J. 2007;83:575-582.

13. Schulman S, Kearon C. Definition of major bleeding in clinical investigations of antihemostatic medicinal products in non-surgical patients. J Thromb Haemost. 2005;3:692-694.

14. CTEP. Common Terminology Criteria for Adverse Events (CTCAE). Protocol Development. https://ctep.cancer.gov/protocoldevelopment/ electronic_applications/ctc.htm. Accessed November 19, 2020.

15. Wells G, Shea B, O'Connell D, et al. The Newcastle-Ottawa Scale (NOS) for assessing the quality of nonrandomised studies in meta-analyses. http://www.ohri.ca/programs/clinical_epidemiology/oxford.asp. Accessed October 02, 2020.

16. Viechtbauer $\mathrm{W}$. Conducting meta-analyses in $\mathrm{R}$ with the metafor. J Stat Softw. 2010;36:1-48.

17. Balduzzi S, Rücker G, Schwarzer G. How to perform a meta-analysis with R: a practical tutorial. Evid Based Ment Health. 2019;22: 153-160.

18. R Core Team. R: A Language and Environment for Statistical Computing. 2019. https://www.r-project.org/. Accessed October 02, 2020.

19. Schwarzer G, Chemaitelly H, Abu-Raddad LJ, Rücker G. Seriously misleading results using inverse of Freeman-Tukey double arcsine transformation in meta-analysis of single proportions. Res Synth Methods. 2019;10:476-483.

20. Abdillah JN, Hu Q, Chen X, et al. Heparin-induced thrombocytopenia in infants after heart surgery. Thorac Cardiovasc Surg. 2019;67: 21-27.

21. Al-Eidan FAS, Alrawkan S, Alshammary H, Crowther MA. Comparison of argatroban and fondaparinux for the management of patients with isolated heparin-induced thrombocytopenia. Ann Hematol. 2018;97:2055-2059.

22. Al-Rossaies A, Alkharfy KM, Al-Ayoubi F, Al-Momen A. Heparininduced thrombocytopenia: comparison between response to fondaparinux and lepirudin. Int J Clin Pharmacol. 2011;33:997-1001.

23. Ansara AJ, Arif S, Warhurst RD. Weight-based argatroban dosing nomogram for treatment of heparin-induced thrombocytopenia. Ann Pharmacother. 2009;43:9-18.

24. Anusim N, lonescu F, Konde AS, et al. Outcomes in heparin-induced thrombocytopenia managed with direct oral anticoagulants. Blood. 2019;134:4975-4975.

25. Arangalage D, Lepage L, Faille D, et al. Presentation, management and outcome of heparin-induced thrombocytopenia after valvular heart surgery. Eur J Cardiothorac Surg. 2016;50:1132-1138.

26. Ataya A, Fadul R, Tang A, et al. An analysis of the characteristics of lung transplant patients that develop heparin induced thrombocytopenia type II (HIT) after transplant. J Heart Lung Transplant. 2013;32: S268.

27. Bain J, Meyer A. Comparison of bivalirudin to lepirudin and argatroban in patients with heparin-induced thrombocytopenia. Am J Health Syst Pharm. 2015;72:S104-S109.

28. Bates D, Griffin S, Angel B. Clinical experience with argatroban for heparin-induced thrombocytopenia in a large teaching hospital. Can J Hosp Pharm. 2009;62:290-297.

29. Begelman SM, Baghdasarian SB, Singh IM, Militello MA, Hursting MJ, Bartholomew JR. Argatroban anticoagulation in intensive care patients: effects of heart failure and multiple organ system failure. J Intensive Care Med. 2008;23:313-320. 
30. Beiderlinden M, Treschan T, Görlinger K, Peters J. Argatroban in extracorporeal membrane oxygenation. Artif Organs. 2007;31: 461-465.

31. Bhatt SH, Adra M. Evaluation of fondaparinux for the treatment of serotonin release assay positive heparin-induced thrombocytopenia. J Thromb Haemost. 2013;11:541.

32. Boon DMS, Kappers-Klunne MC, Michiels JJ, Stibbe J, van Vliet HHDM. Heparin-induced thrombocytopenia and thrombosis: a potential fatal complication in a routine treatment. Neth J Med. 1995;46:146-152.

33. Boyce SW, Bandyk DF, Bartholomew JR, Frame JN, Rice L. A randomized, open-label pilot study comparing desirudin and argatroban in patients with suspected heparin-induced thrombocytopenia with or without thrombosis: PREVENT-HIT study. Am J Ther. 2011;18: 14-22.

34. Buerger T, Tautenhahn J, Meyer F, Lippert H. Heparin-induced vascular occlusion in vasculosurgical patients. An evaluation of the disease in 13 cases. J Cardiovasc Surg (Torino). 1999;40:237-242.

35. Cegarra-Sanmartín V, González-Rodríguez R, Paniagua-Iglesias P, et al. Fondaparinux as a safe alternative for managing heparininduced thrombocytopenia in postoperative cardiac surgery patients. J Cardiothorac Vasc Anesth. 2014;28:1008-1012.

36. Chong BH, Gallus AS, Cade JF, et al. Prospective randomised openlabel comparison of danaparoid with dextran 70 in the treatment of heparin-induced thrombocytopaenia with thrombosis: a clinical outcome study. Thromb Haemost. 2001;86:1170-1175.

37. Curzio KM, Cheng-Lai A, Kheyfets V, Sinnet M, Billett HH. A comparison of direct thrombin inhibitors in the treatment of heparininduced thrombocytopenia: a single institution experience. J Thromb Thrombolysis. 2009;28:117-123.

38. Davis KA, Davis DO. Direct acting oral anticoagulants for the treatment of suspected heparin-induced thrombocytopenia. Eur J Haematol. 2017;99:332-335.

39. Deitcher SR, Topoulos AP, Bartholomew JR, Kichuk-Chrisant MR. Lepirudin anticoagulation for heparin-induced thrombocytopenia. J Pediatr. 2002;140:264-266.

40. Demma LJ, Paciullo CA, Levy JH. Recognition of heparin-induced thrombocytopenia and initiation of argatroban therapy after cardiothoracic surgery in the intensive care unit. $J$ Thorac Cardiovasc Surg. 2012;143:1213-1218.

41. Duewell BE, Briski MJ, Feih JT, Rinka JRG, Tawil JN. Argatroban versus bivalirudin in the treatment of suspected or confirmed heparininduced thrombocytopenia. J Pharm Pract. 2019; 089719001988286. https://doi.org/10.1177/0897190019882866. [Epub ahead of print].

42. Dulicek P, Ivanova E, Kostal M, Fiedlerova Z, Sadilek P, Hirmerova J. Heparin-induced thrombocytopenia treated with fondaparinux: single center experience. Int Angiol. 2020;39:76-81.

43. Faille D, Bourrienne M-C, Gonthier M-C, Huisse MG, Gkalea V, Ajzenberg N. Fibrin monomers to monitor anticoagulation with argatroban in patients with acute heparin induced thrombocytopenia. ISTH Acad. 2019;26365:PB0379.

44. Farasatinasab M, Zarei B, Moghtadaei M, Nasiripour S, Ansarinejad N, Zarei M. Rivaroxaban as an alternative agent for heparin-induced thrombocytopenia. J Clin Pharmacol. 2020;60: 1362-1366.

45. Farner B, Eichler P, Kroll H, Greinacher A. A comparison of danaparoid and lepirudin in patients with heparin-induced thrombocytopenia. Infusionsther Transfusionsmed. 2001;28:12-13.

46. Fischer KG, Van Loo ADE, Böhler J. Recombinant hirudin (lepirudin) as anticoagulant in intensive care patients treated with continuous hemodialysis. Kidney Int Suppl. 1999;56:46-50.

47. Farsad F, Foroughinia F, Gholami K, Ahmadi S. Usefulness of danaparoid sodium in patients with heparin-induced thrombocytopenia after cardiac surgery. J Res Pharm Pract. 2015;4:73-78.
48. Freeman T, Khorsid S, Retter A. Retrospective review of argatroban use and dosing in critical care. Crit Care. 2018;22:82.

49. Freeman W, Bay C, Sharifi M, Breed C, Schwartz F. Treatment of heparin-induced thrombocytopenia with new oral anticoagulants. J Am Coll Cardiol. 2014;63(suppl 12):A2096.

50. Chiquito Freile MT, Hidalgo FN, Martinez Molina JA, Lecumberri R, Figuerola Rosselló A, Medrano TP. Fondaparinux treatment in patients with acute heparin-induced thrombocytopenia and acute renal failure with continuous renal replacement therapy. Intensive Care Med Exp. 2016;4:A453.

51. Gindre L, de Maistre E, Perrin A, Lecompte T, Hoffman MA. Thrombocytopenia due to heparin therapy. Use of danaparoid (Orgaran), 13 monocentric cases under authorization of temporary prescription (ATU). Therapie. 1997;52:591-597.

52. Grouzi E, Kyriakou E, Panagou I, Spiliotopoulou I. Fondaparinux for the treatment of acute heparin-induced thrombocytopenia: a single-center experience. Clin Appl Thromb Hemost. 2010;16: 663-667.

53. Grzegorczyk F, Kuca P, Dybowska M, et al. Efficacy assessment of fondaparinux treatment of patients with hitheparin induced thrombocytopenia (HIT). J Thromb Haemost. 2015;13:865.

54. Hartman V, Malbrain M, Daelemans R, Meersman P, Zachée P. Pseudo-pulmonary embolism as a sign of acute heparin-induced thrombocytopenia in hemodialysis patients: safety of resuming heparin after disappearance of HIT antibodies. Nephron Clin Pract. 2006; 104:143-148.

55. Hoffman WD, Czyz Y, McCollum DA, Hursting MJ. Reduced argatroban doses after coronary artery bypass graft surgery. Ann Pharmacother. 2008;42:309-316.

56. Huhle G, Hoffmann U, Song X, Wang LC, Heene DL, Harenberg J. Immunologic response to recombinant hirudin in HIT type II patients during long-term treatment. Br J Haematol. 1999;106:195-201.

57. Jang IK, Baron SJ, Hursting MJ, Anglade E. Argatroban therapy in women with heparin-induced thrombocytopenia. J Womens Health (Larchmt). 2007;16:895-901.

58. Joseph L, Casanegra Al, Dhariwal M, et al. Bivalirudin for the treatment of patients with confirmed or suspected heparin-induced thrombocytopenia. J Thromb Haemost. 2014;12:1044-1053.

59. Kang M, Alahmadi M, Sawh S, Kovacs MJ, Lazo-Langner A. Fondaparinux for the treatment of suspected heparin-induced thrombocytopenia: a propensity score-matched study. Blood. 2015; 125:924-929.

60. Keren-Politansky A, Hoffman R, Nadir Y, Brenner B, Sarig G. Experience in diagnostic assays for heparin-induced thrombocytopeniaexperience of a tertiary hospital in Israel. Harefuah. 2019;158(3): 160-164.

61. Kim SC, Tran N, Schewe JC, et al. Safety and economic considerations of argatroban use in critically ill patients: a retrospective analysis. J Cardiothorac Surg. 2015;10:1-8.

62. Kiser TH, Burch JC, Klem PM, Hassell KL. Safety, efficacy, and dosing requirements of bivalirudin in patients with heparin-induced thrombocytopenia. Pharmacotherapy. 2008;28:1115-1124.

63. Kiser TH, Jung R, MacLaren R, Fish DN. Evaluation of diagnostic tests and argatroban or lepirudin therapy in patients with suspected heparin-induced thrombocytopenia. Pharmacotherapy. 2005;25: 1736-1745.

64. Kodityal S, Nguyen PH, Kodityal A, Sherer J, Hursting MJ, Rice L. Argatroban for suspected heparin-induced thrombocytopenia: contemporary experience at a large teaching hospital. J Intensive Care Med. 2006;21:86-92.

65. Kunk PR, Brown J, McShane M, Palkimas S, Gail MB. Direct oral anticoagulants in hypercoagulable states. J Thromb Thrombolysis. 2017;43:79-85.

66. Kuo KHM, Kovacs MJ. Fondaparinux: a potential new therapy for HIT. Hematology. 2005;10:271-275. 
67. Lewis BE, Wallis DE, Hursting MJ, Levine RL, Leya F. Effects of argatroban therapy, demographic variables, and platelet count on thrombotic risks in heparin-induced thrombocytopenia. Chest. 2006; 129:1407-1416.

68. Li JF, Wu L, Wen GY, et al. Platelet count trends and response to fondaparinux in a cohort of heparin-induced thrombocytopenia suspected patients after pulmonary endarterectomy. J Thromb Thrombolysis. 2021;51(3):703-710.

69. Lindhoff-Last E, Betz C, Bauersachs R. Use of a low-molecularweight heparinoid (danaparoid sodium) for continuous renal replacement therapy in intensive care patients. Clin Appl Thromb Hemost. 2001;7:300-304.

70. Linkins L, Warkentin TE. Heparin-induced thrombocytopenia: realworld issues. Semin Thromb Hemost. 2011;37:653-663.

71. Linkins LA, Warkentin TE, Pai M, et al. Rivaroxaban for treatment of suspected or confirmed heparin-induced thrombocytopenia study. J Thromb Haemost. 2016;14:1206-1210.

72. Lobo B, Finch C, Howard A, Minhas S. Fondaparinux for the treatment of patients with acute heparininduced thrombocytopenia. Thromb Haemost. 2008;99:208-214.

73. Lubenow N, Warkentin TE, Greinacher A, et al. Results of a systematic evaluation of treatment outcomes for heparin-induced thrombocytopenia in patients receiving danaparoid, ancrod, and/or coumarin explain the rapid shift in clinical practice during the 1990s. Thromb Res. 2006;117:507-515.

74. Lubenow N, Eichler P, Lietz T, Greinacher A. Lepirudin in patients with heparin-induced thrombocytopenia - results of the third prospective study (HAT-3) and a combined analysis of HAT-1, HAT-2, and HAT-3. J Thromb Haemost. 2005;3:2428-2436.

75. Mahlfeld K, Böck M, Franke J, et al. Beitrag zur bedeutung und inzidenz der heparininduzierten thrombozytopenie (HIT) typ II: eine prospektive studie. Zentralbl Chir. 2001;126:39-43.

76. Mokni W, Aatmani ATDK, Thiranos JC, Koffel JC, Beretz L. Étude rétrospective de l'utilisation du danaparoïde sodique aux hôpitaux universitaires de Strasbourg. J Pharm Clin. 2006;25:138-146.

77. Mudaliar JH, Liem TK, Nichols WK, Spadone DP, Silver D. Lepirudin is a safe and effective anticoagulant for patients with heparinassociated antiplatelet antibodies. J Vasc Surg. 2001;34:17-20.

78. Mukherjee A, Patel A, Ensor J, Ibrahim IF, Baker KR, Rice L. Heparininduced thrombocytopenia with very high antibody titer is associated with slower platelet recovery. Blood. 2017;130(suppl 1):3623.

79. Nasiripour S, Saif M, Farasatinasab M, et al. Dabigatran as a treatment option for heparin-induced thrombocytopenia. J Clin Pharmacol. 2019;59:107-111.

80. Ong SY, Chin YA, Than H, et al. Rivaroxaban for heparin-induced thrombocytopenia: adding to the evidence. Ann Hematol. 2017;96: 525-527.

81. Pendleton R, Wheeler MM, Rodgers GM. Argatroban dosing of patients with heparin-induced thrombocytopenia and an elevated aPTT due to antiphospholipid antibody syndrome. Ann Pharmacother. 2006; 40:972-976.

82. Saini H, Wong G, Huh J-H, Brien B, Murphy L, Woods A. Evaluating efficacy, safety and compliance of argatroban dosing nomogram at Canadian teaching hospitale. J Thromb Haemost. 2015;13:605.

83. Samir J, Thachil J. Argatroban for the prevention and treatment of thrombosis in suspected heparin-induced thrombocytopenia at a specialist tertiary Centre. Br J Haematol. 2018;181:5-211.

84. Schenk JF, Berg G, Mörsdorf S, et al. Heparin-induced thrombocytopenia: a critical risk/benefit analysis of patients in intensive care treated with r-hirudin. Clin Appl Thromb Hemost. 2000;6: 151-156.

85. Schenk JF, Pindur G, Stephan B, et al. On the prophylactic and therapeutic use of danaparoid sodium (Orgaran) in patients with heparininduced thrombocytopenia. Clin Appl Thromb Hemost. 2003;9:25-32.
86. Schiele F, Vuillemenot A, Kramarz $P$, et al. Use of recombinant hirudin as antithrombotic treatment in patients with heparin-induced thrombocytopenia. Am J Hematol. 1995;50:20-25.

87. Schindewolf $M$, Steindl J, Beyer-Westendorf J, et al. Use of fondaparinux off-label or approved anticoagulants for management of heparin-induced thrombocytopenia. J Am Coll Cardiol. 2017;70: 2636-2648.

88. Serasli E, Antoniadou M, Tsara V, et al. Successful management of acute thromboembolic disease complicated with heparin induced thrombocytopenia type II (HIT II): a case series. Thromb J. 2008;6: 1-5.

89. Sharifi M, Bay C, Vajo Z, Freeman W, Sharifi M, Schwartz F. New oral anticoagulants in the treatment of heparin- induced thrombocytopenia. Thromb Res. 2015;135:607-609.

90. Skrupky LP, Smith JR, Deal EN, et al. Comparison of bivalirudin and argatroban for the management of heparin-induced thrombocytopenia. Pharmacotherapy. 2010;30:1229-1238.

91. Smith K, Zemrak W, Savic M, Abramson S, Riker R, Fraser G. 438: Prospective evaluation of a bivalirudin dosing nomogram for heparin-induced thrombocytopenia. Crit Care Med. 2014;42(12): A1465.

92. Smythe MA, Koerber JM, Forsyth LL, Priziola JL, Balasubramaniam M, Mattson JC. Argatroban dosage requirements and outcomes in intensive care versus non-intensive care patients. Pharmacotherapy. 2009;29:1073-1081.

93. Sturtevant JM, Pillans PI, Mackenzie F, Gibbs HH. Heparin-induced thrombocytopenia: recent experience in a large teaching hospital. Intern Med J. 2006;36:431-436.

94. Tardy B, Lecompte T, Boelhen F, et al. Predictive factors for thrombosis and major bleeding in an observational study in 181 patients with heparin-induced thrombocytopenia treated with lepirudin. Blood. 2006;108:1492-1496.

95. Tardy-Poncet B, Tardy B, Reynaud J, et al. Efficacy and safety of danaparoid sodium (ORG 10172) in critically ill patients with heparin-associated thrombocytopenia. Chest. 1999;115: 1616-1620.

96. Tardy-Poncet B, Nguyen P, Thiranos JC, et al. Argatroban in the management of heparin-induced thrombocytopenia: a multicenter clinical trial. Crit Care. 2015;19:396. https://doi.org/10.1186/ s13054-015-1109-0.

97. Tomicic TM, Jagnjic JS, Kruhonja-Galic KGZ, et al. Evaluation of the use of national guidelines for diagnosis and management of heparininduced thrombocytopenia (HIT) in the croatian institute of transfusion medicine in the period from 2011 to 2014. Vox Sang. 2015; 109:310.

98. Trehel Turssis V, Louvain V, Doubine S, Stephan F. Heparin-induced thrombocytopenia in a surgical intensive care unit. In: C44. Cardiopulmonary Interactions and Non-Pulmonary Critical Care. American Thoracic Society, 2010:A4559.

99. Tschudi M, Lämmle B, Alberio L. Dosing lepirudin in patients with heparin-induced thrombocytopenia and normal or impaired renal function: a single-center experience with 68 patients. Blood. 2009; 113:2402-2409.

100. Tsu LV, Dager WE. Comparison of bivalirudin dosing strategies using total, adjusted, and ideal body weights in obese patients with heparin-induced thrombocytopenia. Pharmacotherapy. 2012;32: 20-26.

101. Vo QAT, Lin JK, Tong LM. Efficacy and safety of argatroban and bivalirudine in patients with suspected heparin-induced thrombocytopenia. Ann Pharmacother. 2015;49:178-184.

102. Walker EA, Roberts AJ, Louie EL, Dager WE. Bivalirudin dosing requirements in adult patients on extracorporeal life support with or without continuous renal replacement therapy. ASAIO J. 2019;65: 134-138. 
103. Warkentin TE, Pai M, Linkins LA. Direct oral anticoagulants for treatment of HIT: update of Hamilton experience and literature review. Blood. 2017;130:1104-1113.

104. Warkentin TE, Pai M, Sheppard JI, Schulman S, Spyropoulos AC, Eikelboom JW. Fondaparinux treatment of acute heparin-induced thrombocytopenia confirmed by the serotonin-release assay: a 30-month, 16-patient case series. J Thromb Haemost. 2011;9:23892396.

105. Wester JPJ, Haas FJLM, Biesma DH, Leusink JA, Veth G. Thrombosis and hemorrage in heparin-induced thrombocytopenia in seriously ill patients. Intensive Care Med. 2004;30:1927-1934.

106. Wester JPJ, Leyte A, Oudemans-van Straaten HM, et al. Low-dose fondaparinux in suspected heparin-induced thrombocytopenia in the critically ill. Neth J Med. 2007;65:101-108.

107. Yoon JH, Yeh RW, Nam KH, Hoffman WD, Agnihotri AK, Jang IK. Safety and efficacy of the argatroban therapy during the early postcardiac surgery period. J Thromb Thrombolysis. 2010;30:276-280.

108. Young G, Boshkov LK, Sullivan JE, et al. Argatroban therapy in pediatric patients requiring nonheparin anticoagulation: an open-label, safety, efficacy, and pharmacokinetic study. Pediatr Blood Cancer. 2011;56:1103-1109.

109. Young SK, Riding D, Antonio ME, Al-Mondhiry HA. Efficacy of a nurse-managed argatroban dose-adjustment protocol in a university teaching hospital. Hosp Pharm. 2012;47:712-720.

110. Yumoto T, Sato K, Fujii N, et al. Three cases of heparin-induced thrombocytopenia associated with polytrauma. Acute Med Surg. 2016;3:46-49.

111. Zöhrer B, Zenz W, Rettenbacher A, et al. Danaparoid sodium (Orgaran) in four children with heparin-induced thrombocytopenia type II. Acta Paediatr. 2001;90:765-771.

112. Sun Z, Lan X, Li S, Zhao H, Tang Z, Xi Y. Comparisons of argatroban to lepirudin and bivalirudin in the treatment of heparin-induced thrombocytopenia: a systematic review and meta-analysis. Int J Hematol. 2017;106:476-483.

113. Bhatt V, Dahal S, Verma V, et al. Fondaparinux for management of heparin-induced thrombocytopenia after cardiovascular intervention: a systematic review. Cardiovasc Hematol Agents Med Chem. 2015;13: 82-86.

114. Shatzel JJ, Crapster-Pregont M, Deloughery TG. Non-vitamin K antagonist oral anticoagulants for heparin-induced thrombocytopenia. A systematic review of 54 reported cases. Thromb Haemost. 2016;116:397-400.

115. Borenstein M, Hedges LV, JPT H, Rothstein HR. Introduction to Meta-Analysis. John Wiley \& Sons, Ltd: Chichester, UK; 2009.

116. Boyce SW. Challenges to the design and execution of controlled clinical studies of anticoagulants in patients with heparin-induced thrombocytopenia: lessons learned. J Thromb Thrombolysis. 2012;33: 124-128.

\section{SUPPORTING INFORMATION}

Additional supporting information may be found online in the Supporting Information section at the end of this article.

How to cite this article: Nilius H, Kaufmann J, Cuker A,

Nagler M. Comparative effectiveness and safety of anticoagulants for the treatment of heparin-induced thrombocytopenia. Am J Hematol. 2021;96:805-815. https:// doi.org/10.1002/ajh.26194 